\title{
Diversity, distribution and assemblage structure of fishes in streams of southern Western Ghats, India
}

\author{
J.A. Johnson ${ }^{1} \&$ M. Arunachalam ${ }^{2}$ \\ ${ }^{1}$ Wildlife Institute of India, P.Box No. 18, Chandrabani, Dehradun, Uttarakhand 248001, India \\ ${ }^{2}$ Sri Paramakalyani Centre for Environmental Sciences, Manonmaniam Sundaranar University, Alwarkurichi, Tamil Nadu 627412 , India \\ Email: ${ }^{1}$ jajohny@ rediffmail.com
}

\begin{abstract}
Diversity, distribution and assemblage structure of fishes were studied in 10 selected streams of southern Western Ghats. The sampling was performed between April 2001 and March 2002. Sixty species of primary freshwater fishes belonging to four orders, 13 families and 27 genera were recorded from the study area. Cyprinids were the most dominant assemblage members in all study streams. Maximum number of species, number of individuals and cyprinids were recorded from Thalayanai stream. More specialized forms Homaloptera santhamparaiensis, Glyptothorax madraspatanum, Noemacheilus guentheri, N. keralensis, N. semiarmatus and $N$. triangularis were recorded in Panniyar stream. High diversity was observed in Achankoil stream. Evenness index of similarity was uniform in all study streams. Similarity cluster analysis showed streams from nearby basins had similar faunal assemblages. Principal Component Analysis was performed to study the similarity of fish assemblages between the study streams. The analysis described clear pattern of segregation between Thalayanai and Karaiyar (east flowing) and Kallar and Achankoil (west flowing) streams. Thirty-nine Western Ghats endemic fishes were recorded from the study area. Current distribution and threats to endemic fishes are discussed.
\end{abstract}

Keywords: Assemblage structure, endemic fishes, fish diversity, species richness, Western Ghats.

Date of publication (online): 26 October 2009 Date of publication (print): 26 October 2009 ISSN 0974-7907 (online) | 0974-7893 (print)

Editor: Neelesh Dahanukar

Manuscript details:

Ms \# 02146

Received 13 February 2009

Final received 16 October 2009

Finally accepted 17 October 2009

Citation: Johnson, J.A. \& M. Arunachalam (2009). Diversity, distribution and assemblage structure of fishes in streams of southern Western Ghats, India. Journal of Threatened Taxa 1(10): 507-513.

Copyright: ( ) J.A. Johnson \& M. Arunachalam 2009. Creative Commons Attribution 3.0 Unported License. JoTT allows unrestricted use of this article in any medium for non-profit purposes, reproduction and distribution by providing adequate credit to the authors and the source of publication.

Author Details: see end of this article.

Author Contributions: see end of this article.

Acknowledgements: JAJ is grateful to the Department of Science and Technology, New Delhi for the financial support under Fast Track Young Scientist scheme. Critical comments from an anonymous reviewer in improving the quality of the manuscript are sincerely appreciated.
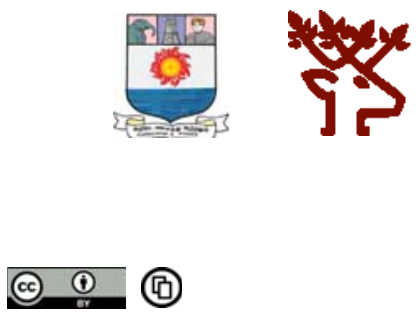

OPEN ACGESS | FREE DOWNLOAD

\section{INTRODUCTION}

Freshwater habitats in rivers, streams, springs and headwaters are heterogeneous due to variations in altitude, flow rates, dissolved oxygen, physical substrate and the riparian zones that provide food, shade and cover (Armantrout 1990). As a result, freshwater habitats harbour diverse fauna, with fish serving as prime indicators of ecosystem status (Karr et al. 1986). Riverine fauna show a high degree of endemism, with most endemic fish species living in headwater streams and/or short stretches of river (Groombridge 1992; Kottelat \& Whitten 1997). Thus riverine freshwater habitats are among the least studied, and likely many species still await discovery (Kottelat \& Whitten 1997).

The Western Ghats biogeographic region of India is home to a highly diverse fish fauna, consisting of 288 known species belonging to 12 orders, 41 families and 109 genera (Dahanukar et al. 2004), of which 116 (53\%) species are endemic to this region (Daniels 2001). Until recently few details on abundance and assemblage organization were available for streams and rivers (Silas 1951; 1953; Rajan 1963; Johnsingh \& Vickram 1987; Devi 1992; Devi \& Menon 1994; Devi et al. 1997; Easa \& Shaji 1997; Johnson \& Soranam 1999; Arunachalam \& Johnson 2002; Arunachalam et al. 2002), however, recent studies have examined diversity distribution and assemblage organization. Arunachalam (2000) studied association of microhabitat variables to species diversity and habitat usage, and diversity, endemism and distribution patterns of fishes have been studied in the central (Bhat, 2003; 2004; Dahanukar et al. 2004) and Kerala regions (Raghavan et al. 2008). While reports on the fish assemblage and distribution patterns seem to cover the Western Ghats fairly well, gaps remain with regard to assemblage structure of the southern region. Thus the main objective of this study is to describe fish assemblage structure in streams of the southern Western Ghats, assess species diversity and similarity of faunal assemblage between streams and evaluate endemism and current conservation status of Western Ghats fish populations.

\section{Materials And Methods}

Study Area: The southern Western Ghats lie between $8^{\circ}-12^{\circ} \mathrm{N} \& 76^{\circ}-78^{\circ} \mathrm{E}$. The region covers about $500 \mathrm{~km}$ of mountain valleys shared by Tamil Nadu and Kerala. The important ecoregions of this area are Agasthyamalai, Anamalai, Cardamom hills and Nilgiris. This hill chain is interrupted in the north by the $30 \mathrm{~km}$ wide Palghat gap at around $11^{\circ} \mathrm{N}$, and south of this gap lies the Anamalai range of hills with its highest peak Anaimudi at $2695 \mathrm{~m}$. This area receives precipitation from both southwest (June to September) and northeast (October to November) monsoons and the average annual precipitation exceeds $2,800 \mathrm{~mm}$. Many torrential and perennial hill streams of this 


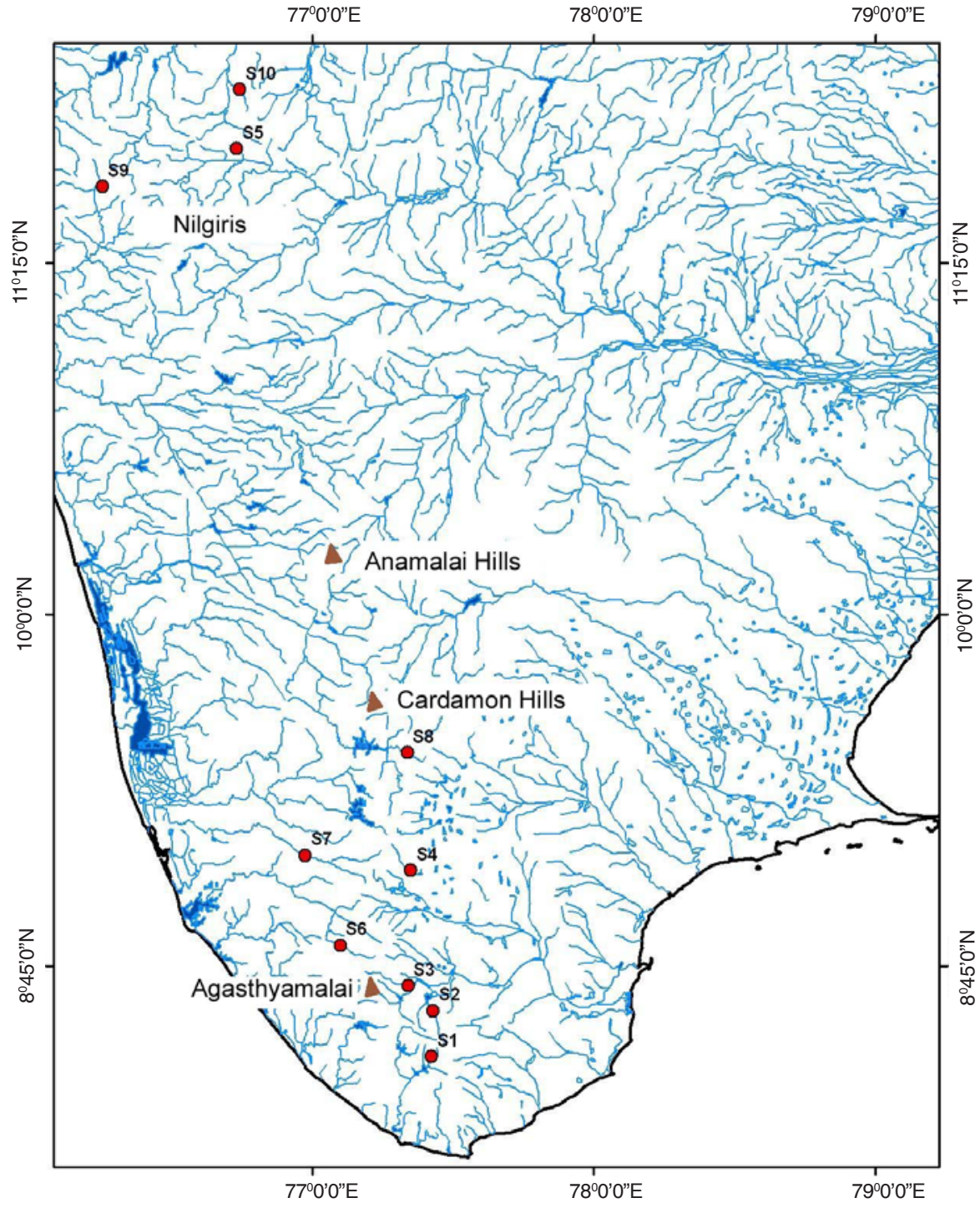

Figure 1. Map indicating sampling sites in southern Western Ghats.

S1 - Samikuchi; S2 - Thalayanai; S3 - Karaiyar; S4 - Hanumannadhi; S5 - Gogalthurai; S6 - Kallar; S7 - Achankoil; S8 - Panniyar; S9 - Thalipuzha; S10 - Bavalipuzha.

region are the main source of major west and east flowing rivers. The western portion is drained by the Vamanapuram, Pamba, Periyar, Bharathapuzha and Chaliyar. In addition to major rivers, number of quick flowing streams originate and quickly discharge into Arabian Sea. The eastern portion is drained by the Tamiraparani, Vaigai, Moyar, Bhavani and Cauvery rivers. In the present study ten streams covering major river basins in Tamil Nadu and Kerala states were selected (Fig. 1) and the sampling was carried out between April 2001 and March 2002. The study streams and their general features are given in Table 1.

Data collection: Fish sampling was performed in $100 \mathrm{~m}$ reach of all study streams. In each stream the sampling was made in different habitats such as pools, riffles, runs and cascades using monofilament gill nets of different mesh sizes ( 10 to $34 \mathrm{~mm}$ ), drag, scoop and cast nets. Fish sampling followed the method of Arunachalam (2000). After collection fishes were examined, counted and released. A few specimens (5-10) of unidentified species were preserved in buffered formalin (10\%) and transported to the laboratory for analysis. Species identification and confirmation were carried out using standard fish taxonomy textbooks (Talwar \& Jhingran 1991; Jayaram 1999). Data on current conservation status was obtained from reports of the Conservation Assessment and Management Plan workshop (Molur \& Walker 1998) on freshwater fishes of India.
Analysis: Information about structure of assemblages was extracted by adopting different univariate indices, namely Margalef's species richness index, Shannon diversity index and Shannon evenness index. The calculation of these indices was followed by the methods of Magurran (1988) and Padhye et al. (2006). Margalef's species richness is calculated using the equation $\mathrm{R}=(\mathrm{S}-1) / \ln \mathrm{N}$, where $\mathrm{S}$ is the number of species, $\mathrm{N}$ is the total number of individuals. The Shannon index of diversity is obtained by the following equation $\mathrm{H}^{\prime}=\Sigma$ pi. In pi, where pi $=$ ni/ $\mathrm{N}$; ni is the number of individuals of 'i'th species and $\mathrm{N}=\sum$ ni. Evenness index is calculated by $\mathrm{E}=\mathrm{H}^{\prime} / \ln \mathrm{S}$, where $\mathrm{S}$ is the number of species. The indices were used to compare the species richness, diversity and equitability across the study streams. The quantitative data of species along with the number of individuals belonging to each species were used to calculate percent similarity index using Bray-Curtis similarity index based on Padhye et al. (2006). Dendrograms were constructed to understand the similarity between the sampling sites. This was done using Bray-Curtis similarity index using nontransformed species abundance data (Anderson 2001). Further, Principal Component Analysis (PCA) was performed to study the similarity of fish assemblage between the study streams and understand which species contributed to the difference in fish assemblage between east and west flowing streams.

\section{Results}

Fish assemblage structure, species diversity and similarity:

During the study a total of 60 species of primary freshwater fishes belonging to four orders, 13 families and 27 genera were recorded from the study streams (Table 2). Number of species, total abundance, cyprinid abundance and their percentage, richness index, Shannon diversity and evenness index for study streams are given in Table 3. Maximum numbers of species and individuals were recorded in Thalayanai stream, while low number of species and individuals were recorded in Hanumannadhi stream. In the assemblage structure, cyprinids constituted the dominant group (72.6 to $92.3 \%)$ and the cyprinids Danio 
Table 1. Summary of study sites characters in southern Western Ghats

\begin{tabular}{|c|c|c|c|c|c|c|c|}
\hline Sites & $\begin{array}{l}\text { River basin East/ } \\
\text { West flowing }\end{array}$ & Latitude/longitude & $\begin{array}{l}\text { Altitude } \\
(\mathrm{m})\end{array}$ & $\begin{array}{l}\text { Stream } \\
\text { order }\end{array}$ & $\begin{array}{l}\text { Stream } \\
\text { gradient } \\
(\%)\end{array}$ & $\begin{array}{l}\text { Mean } \\
\text { width } \\
(\mathrm{m})\end{array}$ & Riparian vegetation \\
\hline Samikuchi (S1) & Chittar - II - West flowing & $8^{\circ} 25^{\prime} 48^{\prime \prime} \mathrm{N} \& 77^{\circ} 25^{\prime} 22^{\prime \prime} \mathrm{E}$ & 500 & 3 & 7 & 45.6 & $\begin{array}{l}50 \% \text { old growth forests and } \\
\text { rubber plantation }\end{array}$ \\
\hline Thalayanai (S2) & Manimuthar - East flowing & $8^{\circ} 35^{\prime} 36^{\prime \prime} \mathrm{N} \& 7^{\circ} 25^{\prime} 42^{\prime \prime} \mathrm{E}$ & 300 & 3 & 7 & 25.6 & $60-70 \%$ old growth forest \\
\hline Karaiyar (S3) & Tamiraparani - East flowing & $8^{\circ} 40^{\prime} 56^{\prime \prime} \mathrm{N} \& 7^{\circ} 20^{\prime} 24^{\prime \prime} \mathrm{E}$ & 300 & 3 & 7 & 13.8 & $70-80 \%$ old growth forest \\
\hline Hanumannadhi (S4) & Chittar - East flowing & $9^{\circ} 05^{\prime} 30^{\prime \prime} \mathrm{N} \& 7^{\circ} 20^{\prime} 59^{\prime \prime} \mathrm{E}$ & 200 & 3 & 2 & 37.4 & $\begin{array}{l}20 \% \text { areca and coconut } \\
\text { farms }\end{array}$ \\
\hline Gugalthurai (S5) & Cauveri - East flowing & $11^{\circ} 40^{\prime} 28^{\prime \prime} N \& 76^{\circ} 45^{\prime} 34^{\prime \prime} \mathrm{E}$ & 600 & 3 & 6 & 11.5 & $60 \%$ old growth forest \\
\hline Kallar (S6) & Vamanapuram - West flowing & $8^{\circ} 45^{\prime} 25^{\prime \prime} \mathrm{N} \& 77^{0} 15^{\prime} 39^{\prime \prime} \mathrm{E}$ & 800 & 3 & 7 & 22 & $70 \%$ old growth forest \\
\hline Achankoil (S7) & Achankoil - West flowing & $9^{0} 10^{\prime} 12^{\prime \prime} \mathrm{N} \& 7^{\circ} 50^{\prime} 28^{\prime \prime} \mathrm{E}$ & 600 & 4 & 4 & 20 & $70-80 \%$ old growth forest \\
\hline Panniyar (S8) & Periyar - West flowing & $9^{\circ} 45^{\prime} 14^{\prime \prime} \mathrm{N} \& 77^{\circ} 15^{\prime} 35^{\prime \prime} \mathrm{E}$ & 912 & 3 & 6 & 14 & $\begin{array}{l}60-70 \% \text { clove and } \\
\text { cardamom plantations }\end{array}$ \\
\hline Thalipuzha (S9) & Cauvery - East flowing & $11^{\circ} 30^{\prime} 51^{\prime \prime} \mathrm{N} \& 76^{\circ} 15^{\prime} 46^{\prime \prime} \mathrm{E}$ & 750 & 3 & 3 & 9.1 & $\begin{array}{l}50 \% \text { areca and orchard } \\
\text { plantation }\end{array}$ \\
\hline Bavalipuzha (S10) & Cauvery - East flowing & $11^{\circ} 55^{\prime} 22^{\prime \prime} N \& 76^{\circ} 45^{\prime} 37^{\prime \prime} E$ & 1350 & 3 & 4 & 20 & $60-70 \%$ old growth forest \\
\hline
\end{tabular}

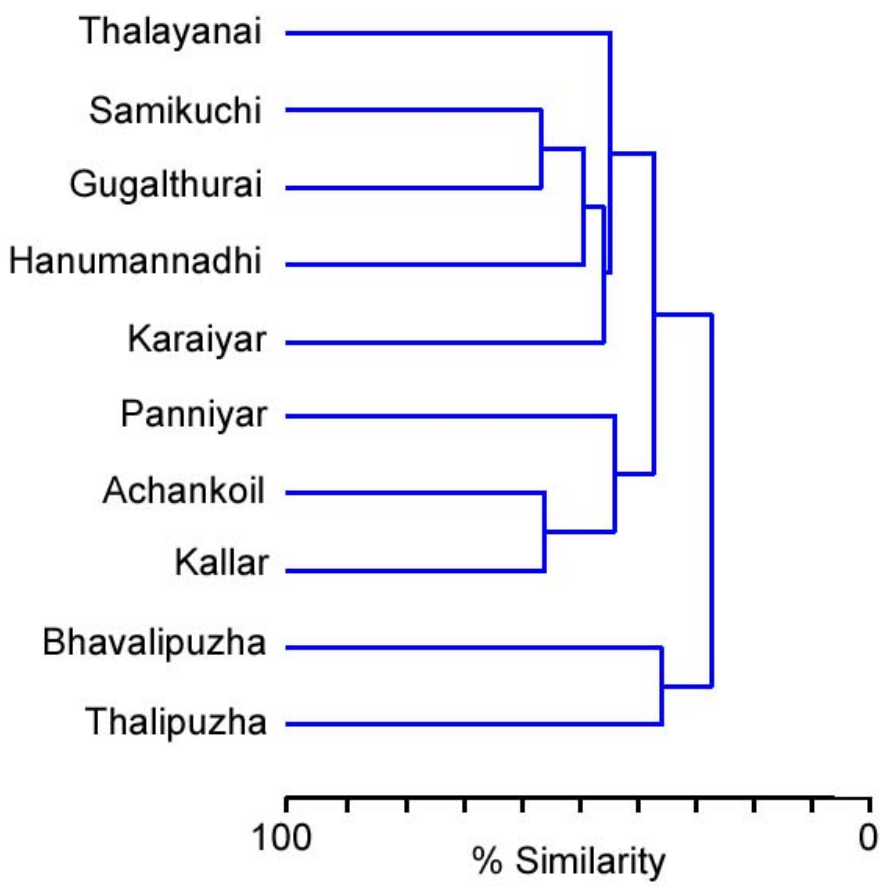

Figure 2. Dendrograms resulting from Bray-Curtis similarities of species abundance data of study streams. [S1-Samikuchi; S2-Thalayanai; S3-Karaiyar; S4-Hanumannadhi; S5-Gogalthurai; S6-Kallar; S7-Achankoil; S8-Panniyar; S9-Thalipuzha; S10Bavalipuzha.]

aequipinnatus, Garra mullya and Rasbora daniconius were represented in all the study streams. The maximum number of cyprinid individuals was recorded from Thalayanai stream, which represented a number of big sized barbs such as Hypselobarbus curmuca, H. kolus, H. dubius, H. dobsoni, Tor khudree and Barbodes sarana. Low cyprinid populations was observed in Bhavalipuzha, followed by Panniyar stream and these streams harboured some specilized forms like Homaloptera santhamparaiensis, Glyptothorax madraspatanum, Noemacheilus guentheri, N. keralensis, N. semiarmatus and N.triangularis. Shannon diversity index showed high value in Achankoil, Kallar and Bhavalipuzha streams. The evenness index of species distribution was uniformly similar in all study streams except Thalayanai stream, where the equitability of species was low.

Cluster analysis of species composition showed that the streams from near by basins had similar faunal assemblage

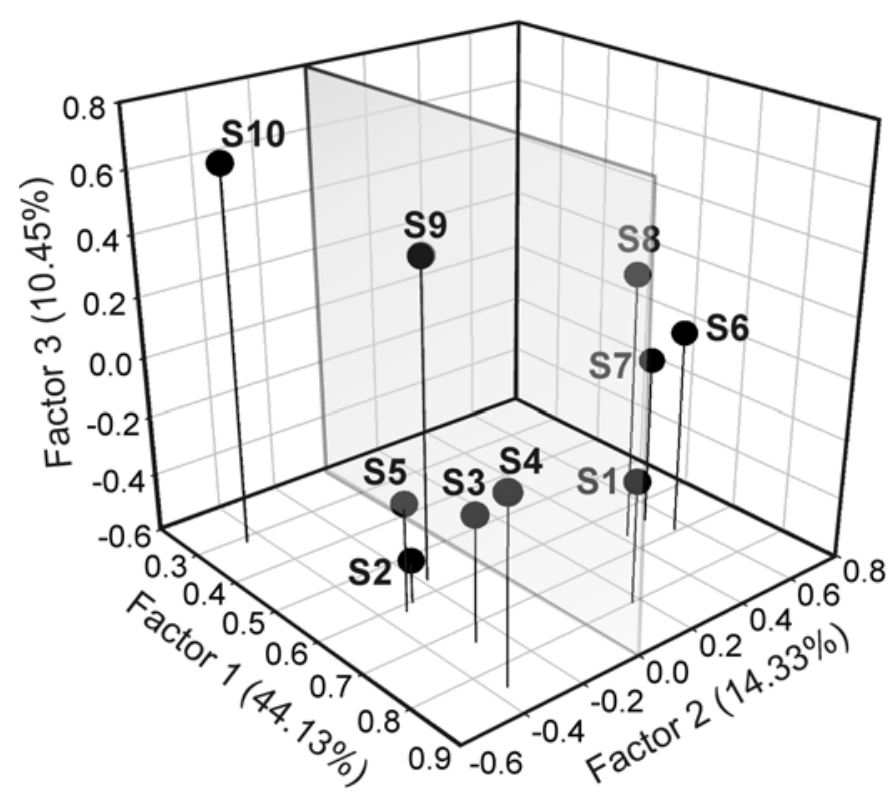

Figure 3. Principal Component Analysis (PCA) of streams based on freshwater fish species assemblage. Only first three significant factors with eigenvalue more than one are plotted. East flowing streams (S2, S3, S4, S5, S9 and S10) separate from the west flowing streams (S1, S6, S7 and S8) on the F2 axis (separated by a grey transparent plane). Numbers in parenthesis are percent variation explained by each PCA factor. Stream numbers are as per Fig. 2.

when the dendrogram was drawn based on the Bray-Curtis similarity index (Fig. 2). Among the east flowing streams, Karaiyar and Thalayanai had more similarity in fish species assemblage. Similarly, in west flowing streams, Bhavalipuzha and Thalipuzha had similar fish assemblage. Principal Component Analysis showed a clear pattern of similarity between the study streams with respect to the fish assemblage (Fig. 3). PCA extracted three significant factors with factor loading above one. These three factors together explained about $70 \%$ of total variation in the data. West flowing rivers (S1, S6, S7 and S8) were separated from the east flowing rivers mainly on the $\mathrm{F} 2$ axis (separated by a transparent plane in Fig. 3). Out of the total 60 fish species, 29 had positive factor loading on $\mathrm{F}_{2}$ axis and were either restricted or had high abundance in the west flowing streams while 31 had negative factor loading on $\mathrm{F}_{2}$ axis and were either restricted or had low abundance to 
Table 2. List of fish species recorded from the study streams of Western Ghats.

\begin{tabular}{|c|c|c|c|c|c|c|c|c|c|c|}
\hline Fish species & $S 1^{*}$ & S2 & S3 & S4 & S5 & S6 & S7 & S8 & S9 & S10 \\
\hline $\begin{array}{l}\text { Cyprinodontiformes } \\
\text { Aplocheilidae }\end{array}$ & & & & & & & & & & \\
\hline Aplocheilus lineatus & - & - & - & - & - & - & 18 & - & 11 & - \\
\hline Aplocheilus panchax & - & - & - & - & - & - & 8 & - & - & - \\
\hline \multicolumn{11}{|l|}{$\begin{array}{l}\text { Cyprinidae } \\
\text { Genus: Barbodes }\end{array}$} \\
\hline Barbodes carnaticus & - & - & - & - & 48 & - & - & - & - & - \\
\hline \multicolumn{10}{|l|}{ Genus: Barilius } & - \\
\hline Barilius bakeri & 42 & - & - & - & - & 72 & 76 & 44 & - & - \\
\hline $\begin{array}{l}\text { Barilius gatensis } \\
\text { Genus: Danio }\end{array}$ & - & - & - & - & 24 & - & - & - & 54 & - \\
\hline Danio aequipinnatus & 42 & 85 & 68 & 88 & 44 & 38 & 34 & 36 & 42 & 28 \\
\hline \multicolumn{10}{|l|}{ Genus: Garra } & 25 \\
\hline Garra gotyla stenorhynchus & - & - & - & - & - & - & - & - & - & 15 \\
\hline Garra hughi & - & - & - & - & - & 15 & - & - & - & - \\
\hline Garra mcclellandi & - & - & - & - & - & - & - & 33 & - & - \\
\hline $\begin{array}{l}\text { Garra mullya } \\
\text { Genus: Horalabiosa }\end{array}$ & \multicolumn{6}{|c|}{ Genus: Horalabiosa } & 46 & 14 & 36 & 42 \\
\hline Horalabiosa arunachalami & - & - & - & - & - & - & - & 11 & - & - \\
\hline \multicolumn{10}{|l|}{ Genus: Hypselobarbus } & - \\
\hline Hypselobarbus curmuca & - & 16 & - & - & - & - & - & - & - & - \\
\hline Hypselobarbus dobsoni & - & 68 & - & - & - & - & - & - & - & - \\
\hline Hypselobarbus dubius & - & 18 & - & - & - & - & - & - & - & - \\
\hline Hypselobarbus kolus & - & 14 & - & - & - & - & - & - & - & - \\
\hline Hypselobarbus kurali & - & - & - & - & - & 26 & - & - & - & - \\
\hline \multicolumn{11}{|l|}{ Genus: Osteochilichthys } \\
\hline \multicolumn{10}{|l|}{ Genus: Puntius } & - \\
\hline Puntius amphibious & - & 68 & - & - & - & - & 55 & - & - & - \\
\hline Puntius arenatus & - & - & - & - & - & - & 32 & - & - & - \\
\hline Puntius tambraparniei & - & 88 & 22 & - & - & - & - & - & - & - \\
\hline Puntius bimaculatus & 64 & 45 & - & 34 & 28 & - & - & - & - & - \\
\hline Puntius conchonius & - & - & - & - & - & - & - & - & 16 & 22 \\
\hline Puntius denisonii & - & - & - & - & - & - & 24 & - & - & - \\
\hline Puntius dorsalis & 32 & 22 & - & - & 38 & - & 38 & - & - & - \\
\hline Puntius fasciatus & - & - & - & - & - & 35 & - & - & - & - \\
\hline Puntius filamentosus & 22 & 38 & 24 & - & 14 & 56 & 52 & - & - & - \\
\hline Puntius kannikattiensis & - & - & 22 & - & - & - & - & - & - & - \\
\hline Puntius melanampyx & 32 & - & - & - & - & 65 & 46 & 45 & 32 & - \\
\hline Puntius parrah & - & - & - & - & - & - & - & - & - & 34 \\
\hline Puntius sophore & - & - & - & - & - & - & - & - & - & 24 \\
\hline \multicolumn{11}{|l|}{ Genus: Rasbora } \\
\hline Rasbora caverii & - & - & - & - & - & - & - & - & - & 14 \\
\hline \multicolumn{11}{|l|}{ Genus: Salmostoma } \\
\hline Salmostoma boopis & - & - & - & - & - & - & 15 & - & - & - \\
\hline \multicolumn{11}{|l|}{ Genus: Tor } \\
\hline Tor khudree & 24 & 12 & 37 & - & 23 & 30 & - & - & - & - \\
\hline \multicolumn{11}{|l|}{$\begin{array}{l}\text { Balitoridae } \\
\text { Genus: Bhavania }\end{array}$} \\
\hline $\begin{array}{l}\text { Bhavania australis } \\
\text { Genus: Noemacheilus }\end{array}$ & 12 & 18 & 17 & - & - & 24 & 22 & - & - & - \\
\hline Noemacheilus denisoni & - & - & - & - & - & 15 & - & - & - & - \\
\hline Noemacheilus guentheri & - & - & - & - & - & - & - & 6 & - & - \\
\hline Noemacheilus keralensis & - & - & - & - & - & - & - & 28 & - & - \\
\hline Noemacheilus semiarmatus & - & - & - & - & - & - & - & 15 & 13 & 16 \\
\hline $\begin{array}{l}\text { Noemacheilus triangularis } \\
\text { Genus: Homaloptera }\end{array}$ & 12 & 15 & 10 & 24 & 18 & 28 & - & 25 & - & - \\
\hline $\begin{array}{l}\text { Homaloptera santhamparaiensis } \\
\text { Genus: Botia }\end{array}$ & - & - & - & - & - & - & - & 7 & - & - \\
\hline Botia striata & - & - & - & - & - & 5 & - & - & - & - \\
\hline $\begin{array}{l}\text { Gobitidae } \\
\text { Genus: Lepidocephalus }\end{array}$ & & & & & & & & & & \\
\hline Lepidocephalus thermalis & - & - & - & 14 & 18 & 15 & - & - & - & - \\
\hline $\begin{array}{l}\text { Siluriformes } \\
\text { Bagridae } \\
\text { Genus: Mystus }\end{array}$ & & & & & & & & & & \\
\hline Mystus armatus & - & - & - & - & - & - & - & - & 25 & - \\
\hline
\end{tabular}




\begin{tabular}{|c|c|c|c|c|c|c|c|c|c|c|}
\hline Fish species & $\mathrm{S} 1^{*}$ & S2 & S3 & S4 & S5 & S6 & S7 & S8 & s9 & S10 \\
\hline $\begin{array}{l}\text { Genus: Batasio } \\
\text { Batasio travancoria }\end{array}$ & - & - & - & - & - & - & 10 & - & - & - \\
\hline $\begin{array}{l}\text { Clariidae } \\
\text { Genus: Clarias }\end{array}$ & & & & & & & & & & \\
\hline Clarias dussumieri & - & - & - & - & - & - & - & - & - & 15 \\
\hline $\begin{array}{l}\text { Siluridae } \\
\text { Genus: Silurus } \\
\text { Silurus wynaadensis }\end{array}$ & - & 8 & - & - & - & 8 & - & - & - & - \\
\hline $\begin{array}{l}\text { Sisoridae } \\
\text { Genus: Glyptothorax } \\
\text { Glyptothorax madraspatanum }\end{array}$ & - & 2 & - & - & _ & - & - & 5 & - & - \\
\hline $\begin{array}{l}\text { Schilbeidae } \\
\text { Genus: Proeutropiichthys } \\
\text { Proeutropiichthys taakree taakree }\end{array}$ & - & - & - & - & - & - & 4 & - & - & - \\
\hline $\begin{array}{l}\text { Perchiformes } \\
\text { Ambassidae } \\
\text { Genus: Pseudambassis }\end{array}$ & & & & & & & & & & \\
\hline $\begin{array}{l}\text { Pseudambassis baculis } \\
\text { Pseudambassis ranga }\end{array}$ & - & $\begin{array}{l}- \\
-\end{array}$ & $\begin{array}{l}- \\
-\end{array}$ & - & - & $\begin{array}{l}- \\
-\end{array}$ & $\begin{array}{l}- \\
-\end{array}$ & - & - & $\begin{array}{l}28 \\
24\end{array}$ \\
\hline $\begin{array}{l}\text { Channidae } \\
\text { Genus: Channa } \\
\text { Channa orientalis }\end{array}$ & - & - & - & - & - & 10 & - & - & - & - \\
\hline $\begin{array}{l}\text { Cichlidae } \\
\text { Genus: Etroplus } \\
\text { Etroplus maculates }\end{array}$ & - & - & - & - & - & - & 34 & - & - & - \\
\hline $\begin{array}{l}\text { Mastacembelidae } \\
\text { Genus: Mastacembelus } \\
\text { Mastacembelus armatus }\end{array}$ & - & 4 & - & - & - & - & - & - & - & 10 \\
\hline
\end{tabular}

*Stream numbers as per Table 1.

Table 3. Variation in species abundance, cyprinid abundance, richness index, Shannon index and evenness index

\begin{tabular}{|c|c|c|c|c|c|c|c|c|c|c|}
\hline Study sites & $\mathrm{S} 1^{*}$ & S2 & S3 & S4 & S5 & S6 & S7 & S8 & s9 & S10 \\
\hline Species & 12 & 19 & 10 & 6 & 15 & 17 & 17 & 13 & 11 & 15 \\
\hline Individuals & 354 & 609 & 278 & 239 & 407 & 521 & 568 & 314 & 267 & 330 \\
\hline Cyprinid abundance & 330 & 562 & 251 & 201 & 335 & 396 & 520 & 228 & 226 & 237 \\
\hline Cyprinids Percentage (\%) & 93.2 & 92.3 & 90.3 & 84.1 & 82.3 & 80.0 & 91.6 & 72.6 & 84.6 & 72.0 \\
\hline Shannon index & 2.38 & 2.61 & 2.17 & 1.64 & 2.63 & 2.64 & 2.66 & 2.36 & 2.24 & 2.64 \\
\hline Margalef's index & 1.87 & 2.81 & 1.60 & 0.91 & 2.33 & 2.56 & 2.52 & 2.09 & 1.80 & 2.41 \\
\hline Evenness index & 0.96 & 0.88 & 0.94 & 0.91 & 0.97 & 0.93 & 0.94 & 0.92 & 0.93 & 0.97 \\
\hline
\end{tabular}

*Stream numbers as per Table 1.

east flowing rivers. Out of these, fishes like Puntius conchonius, Danio (Brachydanio) rerio, Garra gotyla stenorhynchus, Mystus armatus and Pseudambassis ranga, which were restricted to the east flowing streams and Puntius denisonii and Etroplus maculates, which were restricted while west flowing streams showed similar pattern to the one documented by Easa \& Shaji (1997). Other fish species which were restricted to east flowing streams were Barbodes carnaticus, Horalabiosa joshuai, Hypselobarbus curmuca, H. dobsoni, H. dubius, H. kolus, H. micropogon, Puntius kannikattiensis, P. parrah, Rasbora caverii and Salmostoma clupeoides and those restricted to the west flowing streams were Hypselobarbus kurali, Puntius fasciatus, Puntius arenatus, Homaloptera santhamparaiensis, Noemacheilus keralensis and Batasio travancoria.

\section{Endemism and current conservation status:}

Out of 60 species, 39 species are endemic to Western Ghats of Peninsular India (Table 4). Among the endemic species nine are strictly endemic to west flowing systems of southern Western Ghats (Barilius bakeri, Batasio travancoria, Gara hughi, Horalabiosa arunachalami, Homaloptera santhamparaiensis, Hypselobarbus kurali, Puntius denisonii, Noemacheilus guentheri and
$N$. keralensis) and five are endemic to east flowing system of southern Western Ghats (Garra gotyla stenorhynchus, Horalabiosa joshuai, Hypselobarbus dubius, Puntius tambraparniei and Puntius kannikattiensis). Out of 39 endemic species, 25 (64\%) are in threatened categories (Table 4), of which three species, Puntius tambraparniei, Puntius narayani and Silurus wynaadensis are Critically Endangered; 14 species (Garra gotyla stenorhynchus, Bhavania australis, Garra hughi, Puntius denisonii, $P$. dorsalis, $P$. fasciatus, P. parrah, Hypselobarbus curmuca, H. kolus, H. kurali, H. dubius, H. micropogon, Noemacheilus keralensis and Botia striata) are Endangered; eight species (Barbodes carnaticus, Barilius bakeri, Batasio travancoria, Clarias dussumieri, Glyptothorax madraspatanum, Proeutropiichthys taakree takkee, Tor khudree and Noemacheilus semiarmatus) are in Vulnerable categories (Molur \& Walker 1998).

\section{Discussion}

The Western Ghats streams exhibit high variability in fish assemblages and the assemblage composition is determined by specific ecological conditions. Notable highest diversity of 
Table 4. Conservation status of endemic fish recorded from the study streams

\begin{tabular}{|c|c|c|c|c|}
\hline \multirow[t]{2}{*}{ SNo } & \multirow[t]{2}{*}{ Western Ghats endemic species } & \multirow[t]{2}{*}{ Status* } & \multicolumn{2}{|c|}{ Endemic to } \\
\hline & & & $\begin{array}{l}\text { West } \\
\text { flowing } \\
\text { system }\end{array}$ & $\begin{array}{l}\text { East } \\
\text { flowing } \\
\text { system }\end{array}$ \\
\hline 1. & Barbodes carnaticus & VU & & \\
\hline 2. & Barilius bakeri & VU & + & \\
\hline 3. & Barilius gatensis & $\mathrm{NE}$ & & \\
\hline 4 & Garra gotyla stenorhynchus & EN & & + \\
\hline 5. & Garra hughi & EN & + & \\
\hline 6. & Garra mcclellandi & $\mathrm{NE}$ & & \\
\hline 7. & Horalabiosa arunachalami & $\mathrm{NE}$ & + & \\
\hline 8. & Horalabiosa joshuai & $\mathrm{NE}$ & & + \\
\hline 9. & Hypselobarbus curmuca & EN & & \\
\hline 10. & Hypselobarbus dobsoni & $\mathrm{NE}$ & & \\
\hline 11. & Hypselobarbus dubius & EN & & + \\
\hline 12. & Hypselobarbus kolus & EN & & \\
\hline 13. & Hypselobarbus kurali & EN & + & \\
\hline 14. & Hypselobarbus micropogon & EN & & \\
\hline 15. & Osteochilichthys nashii & NE & & \\
\hline 16. & Puntius tambraparniei & CR & & + \\
\hline 17. & Puntius bimaculatus & NE & & \\
\hline 18. & Puntius denisonii & EN & + & \\
\hline 19. & Puntius dorsalis & EN & & \\
\hline 20. & Puntius fasciatus & EN & & \\
\hline 21. & Puntius kannikattiensis & $\mathrm{NE}$ & & + \\
\hline 22. & Puntius melanampyx & NE & & \\
\hline 23. & Puntius parrah & EN & & \\
\hline 24. & Rasbora caverii & $\mathrm{NE}$ & & \\
\hline 25. & Salmostoma boopis & $\mathrm{NE}$ & & \\
\hline 26. & Tor khudree & VU & & \\
\hline 27. & Bhavania australis & EN & & \\
\hline 28. & Noemacheilus denisoni & NE & & \\
\hline 29. & Noemacheilus guentheri & LRIC & + & \\
\hline 30. & Noemacheilus keralensis & EN & + & \\
\hline 31. & Noemacheilus semiarmatus & VU & & \\
\hline 32. & Noemacheilus triangularis & LRIc & & \\
\hline 33. & Homaloptera santhamparaiensis & $\mathrm{NE}$ & + & \\
\hline 34. & Botia striata & EN & & \\
\hline 35. & Batasio travancoria & VU & + & \\
\hline 36. & Clarias dussumieri & VU & & \\
\hline 37. & Silurus wynaadensis & CR & & \\
\hline 38. & Glyptothorax madraspatanum & VU & & \\
\hline 39. & Proeutropiichthys taakree taakree & VU & & \\
\hline
\end{tabular}

* - Based on Molur \& Walker (1998); CR - Critically Endangered; EN - Endangered; VU - Vulnerable; LRIc - Lower Risk-least concern; NE - Not Evaluated.

species richness and endemic forms are encountered in Thalayanai, Kallar and Achankoil streams of southern Western Ghats. Ali \& Ripley (1983) hypothesized that the southernmost division of the Western Ghats (south of Palghat Gap $8^{\circ}-9^{\circ} \mathrm{N}$ ) seems to be a natural pass which has played a significant role in isolating a variety of organisms on either side for a long period of time. This proposition is supported by diversity patterns in birds (Ali \& Ripley 1983), amphibians (Daniels 1992; Bhatta 1997) and flora (Nayar 1996). Moreover, the high hill ranges (Malabar, Travancore hills of Kerala and Nilgiris, Anamalai, Palani and Tirunelveli hills of Tamil Nadu) are largely restricted to south of $11^{\circ} \mathrm{N}$. Thus it is evident that environmental conditions such as widespread rainfall (both south-west and north-east monsoons) and cooler climate have played important roles in diversity patterns and endemism in the southern Western Ghats (Daniels 1992; Dahanukar et al. 2004). The diversity of fish species observed in the present study is in line with findings for other flora and fauna.

The species richness of river fauna may be dependent on the accessibility of streams (Horwitz 1978). The high species richness streams of Thalayanai and Achankoil are located in well protected areas and less accessible to people. In addition to the stream accessibility, diversity and distribution patterns of freshwater fishes are associated with different sets of environmental gradients that have been well studied in streams of the Western Ghats (Johnson 1999; Arunachalam 2000; Bhat 2003, 2004). In the present study streams from west flowing systems encountered more endemic forms. These endemic fishes are usually well specialized, and their movements along the river may be very limited. For example, the species Puntius denisonii is endemic to Achankoil river, found only in rapids of the upstream region. Moreover, the west flowing streams originate from high hills and have short stretches with series of rapids and pools, and the fauna of rapids are known to have a very high rate of endemism (Easa \& Shaji 1997; Kottelat \& Whitten 1997).

In the present findings, cyprinids dominate the assemblage structure as they occupy all possible habitats in Western Ghats streams due to their high adaptive variability. Four of the recorded species, Danio aequipinnatus, Garra mullya, Puntius filamentosus and P. conchonius have widespread distribution in India (Talwar \& Jhingran 1991; Jayaram 1999) and they are common and abundant species in Western Ghats streams. Such extensive distribution and their common high abundance suggest that most of these species are capable of tolerating a wide range of environmental conditions. Ajithkumar (1997) pointed out that the distribution of hill stream loaches, Bhavania australis, Noemacheilus denisoni, N. guentheri, N. semiarmatus and N. triangularis are restricted to Mysore, Nilgiris and in the central part of Western Ghats. In this study these species are recorded from various streams in the southern Western Ghats (Table 2), showing that they have well established distribution. Moreover, the endangered big sized barbs Hypselobarbus curmuca (known from Krishna, Godavari and Cauvery basin); H. dobsoni (known only from Krishna river basin); H. dubius (small population in Cauvery at Nilgiris); H. kolus (small numbers in Godavari, Krishna and Cauvery) have very restricted distribution and small fragment of population exist in the Western Ghats rivers (Talwar \& Jhingran 1991; Menon 1992; Molur \& Walker 1998; Jayaram 1999). Viable populations of these endangered species are found in the Thalayanai stream, new from the present study. These restricted and patchy distributions of species have led to small and vulnerable populations.

The present work shows remarkable species diversity and endemism in the southern part of the Western Ghats, and the recent description of three new species (Horalabiosa arunachalami, Homaloptera santhamparaiensis and Puntius kannikattiensis) from the study area indicate that our understanding of the diversity of fish in this area is still far from being complete. Moreover, the study also revealed that many species in the study area are being threatened by various human activities. The major activities are habitat modification, sand mining, removal of riparian vegetation, agriculture activities and destructive fishing. Habitat modifications are very common in southern Western Ghats streams where the channels are locally modified for various purposes like extraction of water for drinking, agriculture etc. As a result there is low surface flow water available in the downstream area and which creates threats to many localized species. In addition, increased sedimentation due to removal of riparian vegetation and entry of agricultural runoff causes severe threats to Western Ghats fish diversity. These lead to silt deposition along the stream bed, converting the heterogeneous 
stony substrate into homogenous sandy substrates and ultimately results in loss of spawning habitat for many fish species. Another major threat to freshwater fish is sand mining along the stream channel which leads to formation of more deeper pools and loss of run and riffle habitats. Moreover, along with sand the microbial and other invertebrate communities in the stream bottom also get lost, as a result the resource availability for stream fishes would affect. In addition to that, destructive fishing methods like liming, mixing latex of euphorbia, poisoning in stream pool also bring drastic effects on fish diversity. Special attention is to be given for conservation of these threatened species, especially the highly threatened large barbs of the Western Ghats, through captive breeding and ranching. Furthermore, habitat requirements of many of the threatened and endemic species are poorly understood and more detailed studies are warranted.

\section{References}

Ajithkumar, C.R. (1997). Biodiversity of the Western Ghats in India, with special reference to conservation of its fish fauna. Species (IUCN) 29: $13-14$

Ali, S. \& S.D. Ripley (1983). Handbook of the Birds of India and Pakistan (compact). Oxford University Press, New Delhi, 737pp.

Anderson, M.J. (2001). Permutation tests for univariate or multivariate analysis of variance and regression. Canadian Journal of Fisheries and Aquatic Sciences 58: 626-639.

Armantrout, N.B. (1990). Conservation in developing countries In: Daniel, J.C. \& J.S. Serrao (eds). Problems and prospects, Proceedings of the centenary seminar on the Bombay National History Society, $656 \mathrm{pp}$

Arunachalam, M. (2000). Assemblage structure of stream fishes in the Western Ghats. Hydrobiology 430: 1-31.

Arunachalam, M. \& J.A. Johnson (2002). A new species of Puntius (Hamilton) from Tamiraparani river. Journal of Bombay National History Society 99(3): 474-480.

Arunachalam, M., J.A. Johnson \& K. Rema Devi (2002). Homaloptera santhampariensis, a new species of Balitorid fish (Teleostei: Balitoridae) from a Western Ghats stream of Kerala, India. Acta Zoologica Taiwanica 13(1): 31-37.

Bhat, A. (2003). Diversity and composition of freshwater fishes in the river systems of Central Western Ghats, India. Environmental Biology of Fishes 68: 25-38.

Bhat, A. (2004). Patterns and distribution of freshwater fishes in rivers of Central Western Ghats, India and their association with environmental gradients. Hydrobiologia 529: 83-97.

Bhatta, G. (1997). Caecilian diversity of the Western Ghats: In search of the rare animals. Special section: Biodiversity of Western Ghats. Current Science 73(2): 183-187.

Dahanukar, N., R. Raut \& A. Bhat (2004). Distribution, endemism and threat status of freshwater fishes in the Western Ghats of India. Journal of Biogeography 31(1): 123-136.

Daniels, R.J.R. (1992). Geographical distribution patterns of amphibians in the Western Ghats, India. Journal of Biogeography 19: 521-529.

Daniels, R.J.R. (2001). Endemic fishes of the Western Ghats and the Satpura hypothesis. Current Science 81: 240-244.

Devi K.R., T.J. Indra, M.B. Raghunathan, M.M. Bai \& M.S. Ravichandran (1997). Ichthyofauna of the Tamiraparani river system, Tamil Nadu. Zoos' Print Journal 12(7): 1-2.

Devi K.R. \& A.G.K. Menon (1994). Horalabiosa palaniensis, A new cyprinid fish from Palani Hills, Western Ghats, South India. Journal of Bombay Natural History \& Society 91: 110-111.

Devi K.R. (1992). Fishes of Kalakad Wildlife Sanctuary, Tirunelveli District, Tamil Nadu, India. Records of Indian Museum 92(1-4): 193209.

Easa, P.S. \& C.P. Shaji (1997). Freshwater fish diversity in Kerala part of Nilgiri Biosphere Reserve. Current Science 73(2): $180-182$.

Groombridge, B. (ed.) (1992). Global biodiversity status of earth's living resources. Chapman and Hall, London, 585pp.
Horwitz, R.J. (1978). Temporal variability patterns and the distributional patterns of stream fishes. Ecological Monograph 48: 307-321.

Jayaram, K.C. (1999). The Fresh Water Fishes of Indian Region. Narendra Publication, New Delhi, India, $551 \mathrm{pp}$.

Johnsingh, A.J.T. \& D. Vickram (1987). Fishes of Mundanthurai Wildlife Sanctuary, Tamil Nadu. Journal of Bombay Natural History \& Society 84(3): 526-533

Johnson, J.A. (1999). Diversity and ecological structure of fishes in selected streams/rivers in Western Ghats. PhD, Thesis submitted to Manonmaniam Sundaranar University, Tamil Nadu, 142pp.

Johnson, J.A. \& R. Soranam (2001). A new species of Horalabiosa Silas from a Kerala stream of the western Ghats. Journal of Bombay Natural History Society 98(3): 392-395.

Karr, J.R., K.D. Fausch, P.L. Angermeier, P.R. Yant \& I.J. Schlosser (1986). Assessing biological integrity in running waters : a method and its rationale Ill. Natural History Survey 5: 28.

Kottelat, M. \& T. Whitten (1997). Freshwater biodiversity in Asia with special reference to fishes. The world bank Technical Report No. 343, Washington DC, 59pp.

Magurran, A.E. (1988). Ecological Diversity and Its Measurement. Croom Helm Publication, London, $167 \mathrm{pp}$.

Menon, A.G.K. (1992). Conservation of freshwater fishes of Peninsular India. Final report Ministry of Environment and Forests, 1989-92, India, 136pp.

Molur, S. \& S. Walker (1998). Report of the Conservation Assessment and Management Plan. Workshop on freshwater fishes of India. Zoo outreach Organization/CBSG, Coimbatore, India, 156pp.

Nayar, M.P. (ed.) (1996). "Hot Spots" of Endemic Plants of India, Nepal and Bhutan. Tropical Botanical Garden, Trivandrum, 252pp.

Padhye, A.D., N. Dahanukar, M. Panigankar, M. Deshpande \& D. Deshpande (2006). Seasonal and landscape wise distribution of butterflies in Tamhini, northern Western Ghats, India. Zoos' Print Journal 21: 2175-2181.

Raghavan, R., G. Prasad, A. Ali \& B. Pereira (2008). Fish fauna of River Chalakudy part of Western Ghats biodiversity hotspot (south India) - patterns of distribution, threats and conservation needs. Biodiversity Conservation 17: 3119-3131.

Rajan, S. (1963). Ecology of the fishes of the river Pykara and Moyar (Nilgiris), south India. Proceedings of Indian Academy of Science 58(B): 291-323.

Silas, E.G. (1951). On a collection of fishes from anamalai and Nelliampathi hill ranges (Western Ghats) with notes on its Zoogeographical significance. Journal of the Bombay Natural History Society 49: 670-681.

Silas, E.G. (1953). New fishes from the Western Ghats, with notes on Puntius arulius (Jerdon). Records of Indian Museum 51: 27-38.

Talwar, P.K. \& A.G. Jhingran (1991). Inland Fishes of India and Adjacent Countries - Vol. 1 \& 2. Oxford IBH Publication, New Delhi, 1158pp.

Author Details: Dr. J.A. Johnson, a freshwater fish ecologist, completed his $\mathrm{PhD}$ from Sri Paramakalyani Centre for Environmental Sciences, Manonmaniam Sundaranar University, Alwarkurichi Tamil Nadu. He has a vast knowledge on taxonomy and ecology of freshwater fishes of India. Presently he is working as a Scientist in the Wildlife Institute of India, Dehradun.

Dr. M. Arunachalam is a Professor and Head at Sri Paramakalyani Centre for Environmental Sciences, Manonmaniam Sundaranar University, Alwarkurichi Tamil Nadu. $\mathrm{He}$ is a leading ichthyologist working on taxonomy and ecology of hill stream fishes of Western Ghats and conservation and management of wetlands.

Author Contribution: The study: JAJ works on taxonomy, ecology and conservation of freshwater fishes of India. MA - Supervision of taxonomy and ecology of freshwatter fishes of Western Ghats. Current paper: JAJ - involved in field sampling, data analysis and prepared the manuscript. MA - assisted in field sampling and technical guidance in data analysis. 